\title{
Development of a spin-exchange relaxation free magnetometer with a compact heating system
}

\author{
Hyun Joon Lee ${ }^{1,2}$, Kiwoong Kim ${ }^{2 *}$, Seong-Joo Lee ${ }^{2}$, Chan-Seok Kang ${ }^{2}$, Kwon Kyu Yư ${ }^{2}$, Yong-Ho Lee ${ }^{2}$, and \\ Han Seb Moon ${ }^{1}$
}

${ }^{1}$ Department of Physics, Busan National University, Busan 609-735, Korea

${ }^{2}$ Brain and Congnition Measurement Lab., Korea Research Institute of Standards and Science (KRISS), Daejoen, Korea

\begin{abstract}
Atomic magnetometers based on spin-exchange relaxation free (SERF) regime typically operate with high optical density and low magnetic fields. A SERF magnetometer with a compact heating system for compact and efficient temperature control is presented. A resistive heating system with high frequency alternating current was used to achieve high optical density. The current frequency was carefully chosen to avoid resonance with the atomic magnetometer. This resulted in a compact heating system that also allows independent operation of multiple heating systems. An optical rotation signal was obtainedat $200^{\circ} \mathrm{Cand}$ width was about $3 \mathrm{nTwith}$ pump laser power of $55 \mathrm{~mW}$. Bandwidth of the magnetometer with the compact heater was $40 \mathrm{~Hz}$. Consequently, the compact resistive heating system proved to be a good alternative to the air-flow heating system. Additionally, residual field dependent signalto-noise yielded a noise level of $50 \mathrm{fT} / \mathrm{Hz}^{1 / 2}$ at $10 \mathrm{~Hz}$.
\end{abstract}

Keywords: atomic vapour; magnetometer; SERF;resistive heating system

Citation: Hyun Joon Lee, Kiwoong Kim, Seong-Joo Lee, Chan-Seok Kang, Kwon Kyu Yu, Yong-Ho Lee, and Han Seb Moon, "Development of a spin-exchange relaxation free magnetometer with a compact heating system”, Acta IMEKO, vol. 2, no. 1, article 7, August 2013, identifier: IMEKO-ACTA-02(2013)-01-07

Editors: Paolo Carbone, University of Perugia, Italy; Ján Šaliga, Technical University of Košice, Slovakia; Dušan Agrež, University of Ljubljana, Slovenia

Received January $10^{\text {th }}, 2013$; In final form July $1^{\text {st }}, 2013$; Published August 2013

Copyright: (C) 2013 IMEKO. This is an open-access article distributed under the terms of the Creative Commons Attribution 3.0 License, which permits unrestricted use, distribution, and reproduction in any medium, provided the original author and source are credited

Funding: none reported

Corresponding author: Kiwoong Kim, e-mail: kwkim@kriss.re.kr

\section{INTRODUCTION}

The atomic coherence between the ground states generated by the interaction of laser light with atoms has been applied to interesting topics such as an atomic magnetometer, frequency standards, light storage, quantum memory, and quantum cryptography [1-10]. Detection of a magnetic field with an atomic magnetometer is performed by monitoring the spin precession due to the external field as observing the optical rotation signal. The principal mechanism of an atomic magnetometer can be described by the classical Faraday rotation. Magneto-optical rotation is an optical effect that makes the polarization plane of linearly-polarized light rotated during its propagation through a medium placed in the magnetic field. When the magnetic field is applied along the light propagation direction, we can see this magneto-optical rotation known as the Faraday's effect. The linearly-polarized light can be decomposed into left $\left(\sigma^{-}\right)$and right $\left(\sigma^{+}\right)$circularlypolarized light. In the presence of a longitudinal magnetic field, the Zeeman sublevels are shifted and two circular polarized light beams are resonant on the transitions with different resonance frequencies, satisfying the selection rules. The Zeeman splitting leads to different dispersion of two circularlypolarized components of the linearly-polarized light. Consequently, the phase difference between two components drives the rotation of its polarization plane [8]. By using this phenomenon, Budker et al have achieved the NMOE (Nonlinear Magneto-optic Effects) signal with an effective resonance width of $\gamma \approx 2 \pi \times 1.3 \mathrm{~Hz}$ [9].

In recent reports, the most sensitive atomic magnetometer is the spin-exchange relaxation free (SERF) magnetometer [11]. In the SERF regime, relaxation due to spin-exchange collisions is eliminated where the spin-exchange rate is much greater than the rate of Larmor precession. In Ref. [10] with a SERF magnetometer, operating potassium $(\mathrm{K})$ vapour cell at $200{ }^{\circ} \mathrm{C}$, a sensitivity of $0.54 \mathrm{fT} / \mathrm{Hz}^{1 / 2}$ was achieved.

The SERF magnetometer is operated with a low magnetic field and a high atomic density for a rapid spin-exchange rate. For the high atomic density, most atomic magnetometers with 
SERF regime have used an air flow oven that requires bulky items such as a double-walled oven that perfectly surrounds the cell for preventing air leakage and perturbation of the optical path. Moreover, an air flow system not only responses sensitively to external vibrations but also has a complex valve system to operate with an independent control on multiple parts in the heating system.

In this paper, we present a SERF regime magnetometer with a compact heating system using modulated current. Further, we experimentally and theoretically investigate the rapid spinexchange effect in a low magnetic field. To attain a better understanding of the residual field effects, we analytically calculated the optical rotation spectra using density matrix equations assuming the spin-temperature distribution to be in the ground state of the $\mathrm{D}_{1}$ line of $\mathrm{K}$. The influence of the residual fields on the resonance signal was observed for different magnetic fields, and the bandwidth as a function of the frequency of the external field was measured to estimate the influence of the resistive heating system.

\section{THEORETICAL MODEL}

The density matrix theory was used for the description of the electron spin polarization. The time evolution of the density matrix $\rho$ well describes the interaction of spins with magnetic field. The density matrix equation is as follows $[12,13]$ :

$$
\begin{aligned}
& \frac{d \rho}{d t}=D \nabla^{2} \rho+\frac{[H, \rho]}{i \hbar}+\frac{\varphi(1+4\langle\mathbf{S}\rangle \cdot \mathbf{S})-\rho}{T_{S E}}+\frac{\varphi-\rho}{T_{S D}}+ \\
& +R[\varphi(1+2 \mathbf{s} \cdot \mathbf{S})-\rho],
\end{aligned}
$$

where $\varphi=\rho / 4+\mathbf{S} \cdot \varrho \mathbf{S}$ is the purely nuclear part of the density matrix and $H$ is the Hamiltonian related to the magnetic moment interaction with the magnetic field, $H=a_{b f} \mathbf{I} \cdot \mathbf{S}+g_{s} \mu_{B} \mathbf{B} \cdot \mathbf{S}$, where $a_{h f}$ is the hyperfine constant, $g_{s}$ is the electron's g-factor, $\mu_{B}$ the Bohr magneton, $\mathbf{I}$ the nuclear angular momentum, and $\mathbf{S}$ the spin angular momentum. Here $R$ is the optical pumping rate, $T_{S D}$ is the relaxation time due to spin-destruction collisions, and $\mathbf{s}$ is the optical pumping vector, giving the direction and the degree of circular polarization of the light. We first neglect the diffusion term for a magnetic field $\mathrm{B}$ in parallel to the direction of optical pumping s. Under the condition that the total angular momentum (F) including the nuclear spin (I) and the number of the $\mathrm{K}$ atoms are conserved, we can simplify the density matrix as a spin-temperature distribution by using the Expectation-Maximum algorithm. Deduced results show that the spin evolution in the ground state can be described by Bloch equations for electron spin polarization $\mathbf{P}=\langle\mathbf{s}\rangle / S[11$, 14, 15]:

$$
\frac{d \mathbf{P}}{d t}=D \nabla^{2} \mathbf{P}+\frac{1}{q(P)}\left(g_{s} \mu_{B} \mathbf{P} \times \mathbf{B}+R(\mathbf{s}-\mathbf{P})-\mathbf{P} \Gamma_{p r}\right),
$$

where $q(P)$ is the nuclear slowing down factor, which depends on the hyperfine sublevel distribution of ensembles of $\mathrm{K}$ atoms, and $\Gamma_{p r}$ is the rate of depolarization due to the probe beam. For the explanation of the SERF regime in the steady-state, $q(P)$ depends on the degree of the partial polarization.

\section{EXPERIMENTAL SETUP}

In order to investigate the optical rotation under SERF regime in the $\mathrm{D}_{1}$ line of $\mathrm{K}$ atomic vapour, the experimental apparatus shown in Fig. 1 is used.

The experiment was performed with a glass cell containing $\mathrm{K}$ vapour, 600-Torr He buffer gas to reduce the rate that atoms in the cell diffuse towards its wall, and 15-Torr $\mathrm{N}_{2}$ to improve optical pumping by quenching. The cell was placed inside a three-layer set of the cylindrical mu-metal chamber. The cell consisted of two parts, a main cell and a stem, respectively. The main cell was roughly cubic, with size about $2.5 \mathrm{~cm}$, and was connected to a stem acting as a reservoir. The stem was composed of a specific glass that prevents $\mathrm{K}$ vapour from depositing on the surface of the cell.

The cell was heated up to $200{ }^{\circ} \mathrm{C}$ by the resistive heating method. The resistive heater was operated by the $25 \mathrm{kHz}$ modulated current source. Fig. 2(a) and (b) show an overall diagram and circuit diagram of the heating system, respectively. The sine wave generated by the XR-2206 oscillator with external frequency adjustment was multiplied with the output voltage from a PID temperature controller after passing through the high pass filter. Then the modulated voltage was amplified by the Crown CE1000 amplifier and converted to the modulated current source. The oscillation frequency $f$ is determined by the external timing capacitor $C$, between Pin 5 and 6 of XR-2206, and by the timing resistor R, connected to Pin 7 of the XR-2206. Its oscillation frequency is given by $1 / \mathrm{RC}$ and can be adjusted by varying either $\mathrm{R}$ or $\mathrm{C}$.

In our experimental setup, two independent heaters were used to avoid deposition of the vapour. The temperature differences between the main cell and stem were maintained automatically, most $\mathrm{K}$ atoms could stay in the stem at room temperature. The density of the $\mathrm{K}$ vapour was about $1.4 \times 10^{15}$ $\mathrm{cm}^{-3}$. The resistive heater was insulated by the insulation panel.

The coil system in Fig. 1 consisted of 16 square coils and a Helmholtz coil that eliminates the residual field or generates arbitrary homogeneous fields and gradient fields. When a magnetic field was applied to the $\mathrm{K}$ vapour cell perpendicularly to the pump light propagation, SERF condition was satisfied at near zero magnetic field.

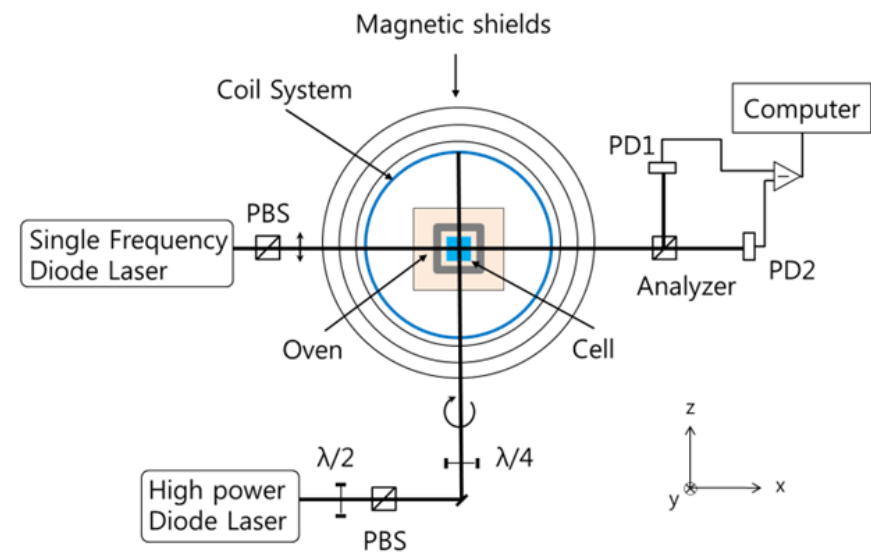

Figure 1. Setup of the SERF magnetometer. Circularly polarized light tuned to the $D_{1}$ line, propagating in the $z$-direction produces ground state orientation in the $z$-direction. Optical rotation of the probe beam is detected using a polarimetric method. (PBS: Polarizing beam splitter, PD: Photo diode, $\lambda / 2$ : Half wave plate, $\lambda / 4$ : Quarter wave plate). 
The distributed feedback (DFB) laser was used in the experiment. The optical pumping was accomplished by circularly polarized laser light propagating in the z-direction, exactly tuned to the center of the $\mathrm{K}_{1}$ line by monitoring the shape of the signal. The pump beam power was amplified from $15 \mathrm{~mW}$ to $150 \mathrm{~mW}$ by a tapered amplifier. The wavelength of the pump laser was monitored by a wavemeter and stabilized on the $\mathrm{K} \mathrm{D}_{1}$ line. To prevent optical feedback we used an optical isolator. We adjusted the laser power by using a polarization beam splitter (PBS) and a half wave plate (HWP). The linearly-polarized probe beam, propagating in the $x$ direction, was detuned from the $\mathrm{K} \mathrm{D}_{1}$ line about several nanometres. The probe beam was generated by a single mode DFB laser and monitored by a Fabry-Perot interferometer. After passing the HWP and the K vapour cell, the laser beam goes through an analyzer which divides the laser beam into two orthogonal directions. The difference between the signals of the photodiodes PD1 and PD2 was measured. The vapour cell was inside a three-layer $\mu$-metal shield that can minimize the effects of the external magnetic field including the Earth's magnetic field. After degaussing, the residual fields inside the shield were of the order of 0.1 to $0.2 \mathrm{nT}$ in the $x$ and $z$-direction and 4 to 5 $\mathrm{n} T$ in the $y$-direction.

\section{EXPERIMENTAL RESULTS}

The sensitivity of the magnetometer depends on the signalto-noise ratio $(\mathrm{S} / \mathrm{N})$ of the Zeeman resonance signal and the linewidth of the resonance. When the optical pumping rate is less than or similar to the spin destruction rate, the linewidth of the resonance is proportional to the transverse spin relaxation time. In this case the sensitivity $\delta \mathrm{B}$ can be written mathematically by

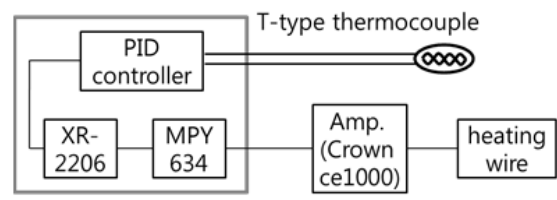

(a)

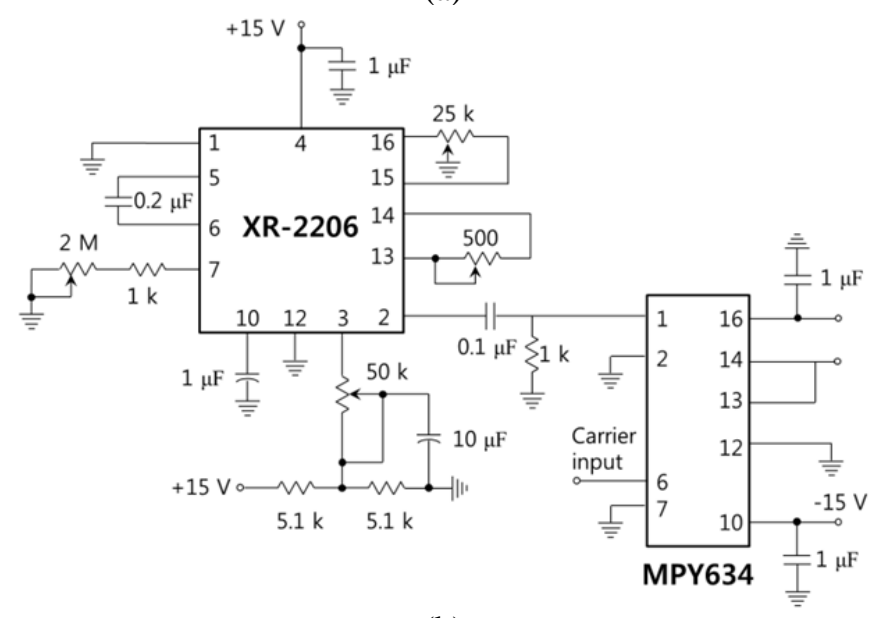

(b)

Figure 2. (a) The schematic diagram of the resistive heating system and (b) the circuit diagram of the high frequency alternating current generator. The XR-2206 generates a sinusoidal waveform voltage which passes through the high pass filter with cut-off frequency $1.5 \mathrm{kHz}$. The sinusoidal wave is multiplied with the carrier input signal (Pin 6 of MPY634) supplied by a PID controller.

$$
\delta B=\frac{\Delta B}{(S / N)},
$$

where $\Delta \mathrm{B}$ is the line width of the magnetic resonance [13]. The sensitivity is commonly regarded as the slope of the curve near resonance. To optimize the magnetometer sensitivity the peakto-peak amplitude and width of the optical rotation signal in the near zero field resonance were observed, for pump powers ranging from $15 \mathrm{~mW}$ to $90 \mathrm{~mW}$. The width was linearly increased according to the pump power (Fig. 3(a)), while the amplitude of the signal appears roughly saturated (fig. 3(b)). In this case the maximum slope $(\partial \varphi / \partial \mathrm{B})$ occurs at $55 \mathrm{~mW}$, as shown in Fig. 3(c). Since the slopes near the resonance provide the relative intrinsic sensitivity of the magnetometer, the system is most sensitive when the pump power is $55 \mathrm{~mW}$. We observed the resonance signals. The dispersive curve shown in Fig. 4 is a typical optical rotation signal as a function of the magnetic field obtained in the atomic system. The spectral width is about $3 \mathrm{nT}$ when the pump beam power was $55 \mathrm{~mW}$. The experimental results show a good agreement with the expression described by Eq. (2). The analytic results were

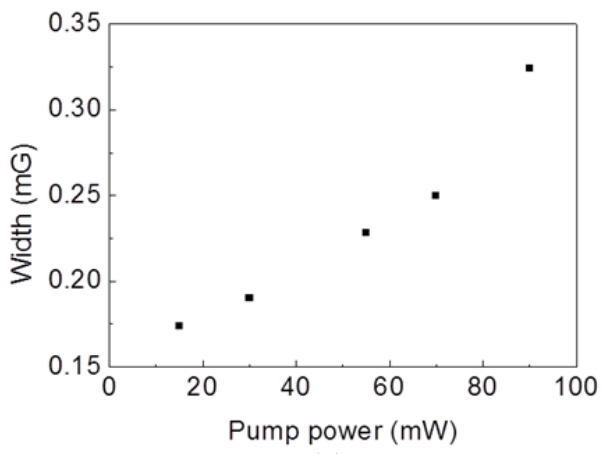

(a)

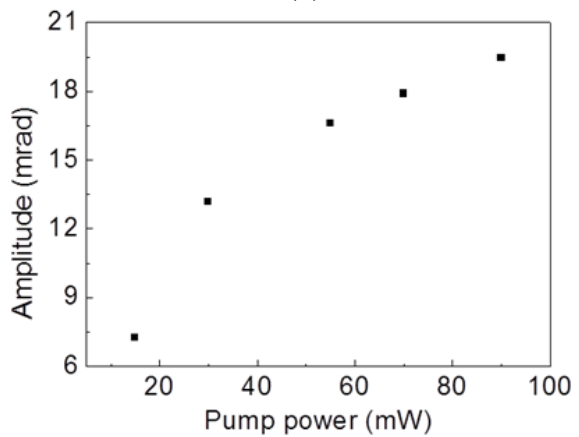

(b)

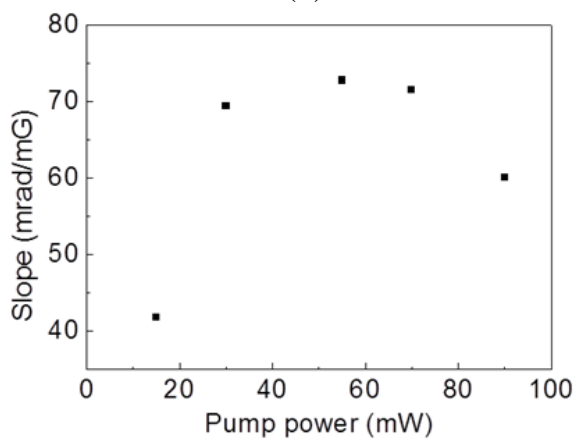

(c)

Figure 3. (a) Half width at half maximum and (b) peak-to-peak amplitude of the zero field resonance were observed to optimize the magnetometer. (c) The magnetometer sensitivity is proportional to the slope of the dispersion curves near resonance. The magnetometer was optimized at $55 \mathrm{~mW}$. 


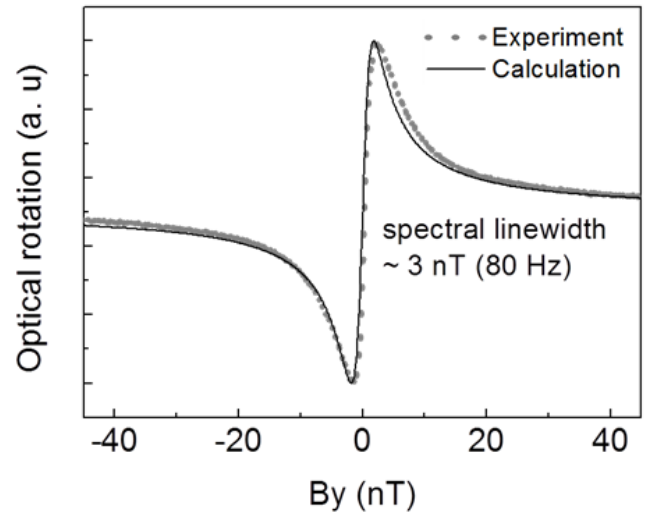

Figure 4. A normalized zero-field resonance spectrum. The horizontal axis in the figure shows the magnetic field in $y$-direction when the other components of the magnetic field were almost nulled. The solid line represents the measurements and the circles represent the values from the expression given by Eq. (2)

obtained as a function of $\mathrm{B}_{\mathrm{y}}$ for all other fields zeroed. The experimental result shows a same linewidth compared to analytic results but an asymmetric curve in the positive magnetic field region. The downward peak was larger than the upward peak of the curve. The analytic calculation shows that this result was caused by the residual magnetic fields in the $x$ and $z$-direction in the same sign.

To investigate the effects of residual fields in the $x$ and $z$ direction in the mu-metal chamber, we applied a bias magnetic field in the $x$ and $z$-direction. Fig. 5 shows the optical rotation signals for several different values of the bias magnetic field. The signal response related to the direction change of the magnetic field is influenced by the magnetic field in the z direction. In Fig. 5(a) and (b), the zero-field resonance signal varied asymmetrically according to the magnetic field in the $x$

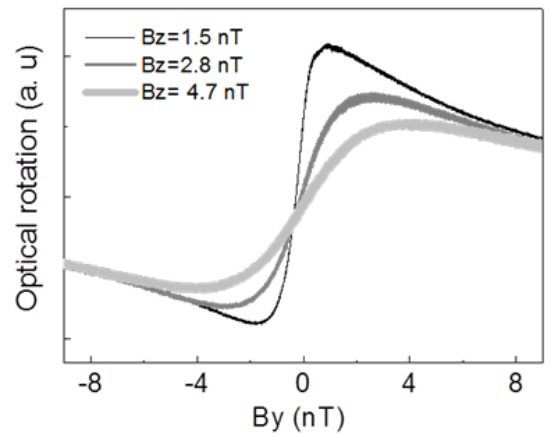

(a)

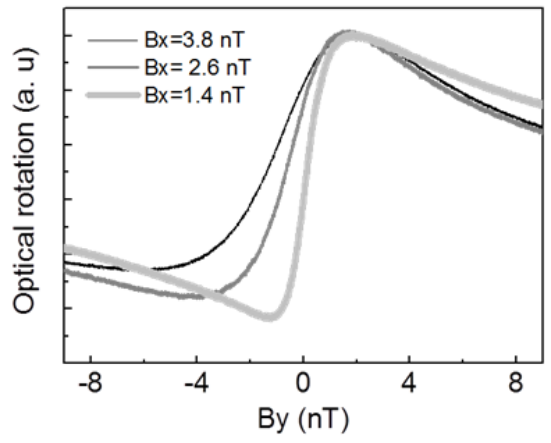

(b)

Figure 5. The effect of DC magnetic fields (a) in the $z$-direction and (b) $x$ direction. and $z$-direction. At increasing magnetic field, the peak-to-peak amplitude of the signal decreased. At the negative magnetic field region the signal change is remarkable. This result means that there is a negative detuned magnetic field in the z-direction. In other words, spin precession transients cannot decay completely before a measurement of the signal is made. The magnetic field dependent signals are the results of the redistribtution of the ground-state populations caused by the magnetic field in the direction of the laser's propagation. The atomic magnetic momentum is arranged to the z-axis, the direction of the pump beam's propagation. However, when there are weak residual fields along the $x$ - and $z$-direction, the atomic magnetic momentum is changed around the zero-field.

To estimate the magnetometer performance, the frequency response and noise level were measured. To calibrate the bandwidth, we apply a small oscillating field with $30 \mathrm{nT}$ at several frequencies in the $y$-direction. The bandwidth of the magnetometer is about $40 \mathrm{~Hz}$ as shown in Fig. 6(a). The result shows that the resistive heating system did not affect the magnetometer because the modulation frequency of the heating system was $24 \mathrm{kHz}$.

We evaluated the performance of the magnetometer by monitoring the noise level at the output of the balanced polarimeter using a spectrum analyzer. To calibrate the magnetometer, we applied a small oscillating field of $7.7 \mathrm{~Hz}$. The resulting spectrum is shown in Fig. 6(b). The powers of the pump and the probe lights were $55 \mathrm{~mW}$ and $3 \mathrm{~mW}$, repectively, and the $y$-component of the magnetic field was zeroed. The sensitivity was about $50 \mathrm{fT} / \mathrm{Hz}^{1 / 2}$ at $10 \mathrm{~Hz}$.

\section{CONCLUSIONS}

We have demonstrated the operation of a magnetometer under SERF regime with a compact heating system. The optical rotation spectra were estimated in the $\mathrm{D}_{1}$ line of $\mathrm{K}$ atoms with

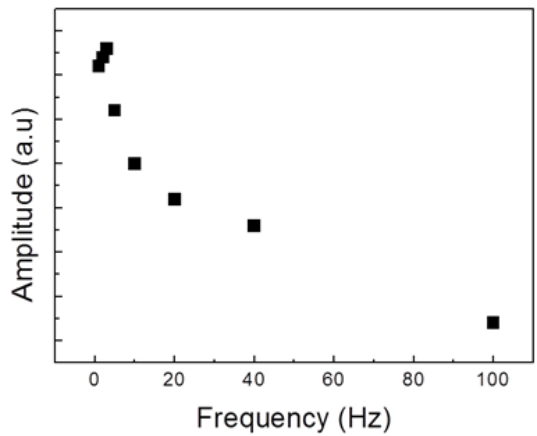

(a)

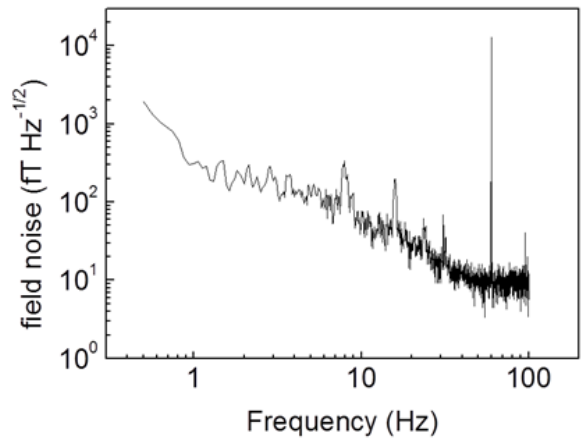

(b)

Figure 6. (a) The frequency response and (b) the noise spectrum of the atomic magnetometer approaching to the SERF regime. 
orthogonal pumping and probing light beams with different power of the pump laser. We have obtained a magneto-optical rotation feature with a linewidth of $3 \mathrm{nT}$. The achieved sensitivity based on optical rotation measurements in the experiment was $50 \mathrm{fT} / \mathrm{Hz}^{1 / 2}$. This value is an order worse than in [10], which is partially explained by the presence of a weak residual field in the magnetic shields. In the SERF regime, the rate of Larmor precession depends on the absolute DC magnetic fields.

We have confirmed that the resistive heating system with high frequency alternating current did not affect the atomic system since the frequency was outside the bandwidth of the magnetometer. Two independent heating systems prevent the atomic vapour from being deposited on the cell window. However, low frequency noise brought into the heating system through the external power line might affect the performance of the magnetometer. Therefore, with adequate measures to prevent inflow of low frequency noise, the resistive heating system could be a practical alternative to air flow heaters.

\section{REFERENCES}

[1] J. Vanier, "Atomic clocks based on coherent population trapping”, Appl. Phys. B, vol. 81, no. 4, 2005, pp. 421-442.

[2] M.D. Lukin, "Trapping and manipulating photon states in atomic ensembles", Rev. Mod. Phys. vol. 75, 2003, pp. 457-472.

[3] C. Liu, Z. Dutton, C.H. Behroozi, L.V. Hau, "Observation of coherent optical information storage in an atomic medium using halted light pulses", Nature, vol. 409, 2001, pp. 490-493.

[4] M. Bajcsy, A.S. Zibrov, M.D. Lukin, "Stationary pulses of light in an atomic medium", Nature, vol. 426, 2003, pp. 638-641.

[5] C. Ottaviani, D. Vitali, M. Artoni, F. Cataliotti, P. Tombesi, "Polarization qubit phase gate in driven atomic media", Phys. Rev. Lett. vol. 90, 2003, pp. 197902-197905.
[6] M. Fleischhauer, M.O. Scully, "Quantum sensitivity limits of an optical magnetometer based on atomic phase coherence", Phys. Rev. A, vol. 49, 1994, pp. 1973-1986.

[7] D. Budker1, D.F. Kimball, S.M. Rochester, V.V. Yashchuk, M. Zolotorev, "Sensitive magnetometry based on nonlinear magneto-optical rotation", Phys. Rev. A, vol. 62, 2000, pp. 043403-043409.

[8] L. Lee, H.S. Moon, J.B. Kim, "The characteristics of nonlinear magneto-optical effects based on coherence population trapping in the $\mathrm{D}_{1}$ line of $\mathrm{Rb}$ atom", Hankook Kwanghak Hoeji, vol. 17, no. 1, 2006, pp. 1-6.

[9] D. Budker, V. Yashchuk, M. Zolotorev, "Nonlinear magnetooptic effects with ultranarrow widths", Phys. Rev. Lett. vol. 81, no. 26, 1998, pp. 5788-5791.

[10] I.K. Kominis, T.W. Kornack, J.C. Allred, M.V. Romalis, “A subfemtotesla multichannel atomic magnetometer", Nature, vol. 422, 2003, pp. 596-599.

[11] J.C. Allred, R.N. Lyman, T.W. Kornack, M.V. Romalis, "Highsensitivity atomic magnetometer unaffected by spin-exchange relaxation", Phys. Rev. Lett., vol. 89, 2002, pp. 130801-130804.

[12] W. Happer, A.C. Tam, "Effect of rapid spin exchange on the magnetic-resonance spectrum of alkali vapors", Phys. Rev. A, vol. 16, 1977, pp. 1877-1891.

[13] S. Appelt, A. Ben-Amar Baranga, C.J. Erickson, M.V. Romalis, A.R. Young, W. Happer, "Theory of spin-exchange optical pumping of ${ }^{3} \mathrm{He}$ and ${ }^{129} \mathrm{Xe}$ ", Phys. Rev. A, vol. 58, 1998, pp. 1412-1439.

[14] S.J. Seltzer, M.V. Romalis, "Unshielded three-axis vector operation of a spin-exchange-relaxation-free atomic magnetometer", Appl. Phys. Lett., vol. 85, 2004, pp. 4804-4806.

[15] M.P. Ledbetter, I.M. Savukov, V.M. Acosta, D. Budker, M.V. Romalis, "Spin-exchange-relaxation-free magnetometry with Cs vapour", Phys. Rev. A, vol. 77, 2008, pp. 033408-033414. 